\title{
Correction to: Mechanical Ventilation in Emergency Medicine
}

\section{Correction to: S. R. Wilcox et al., Mechanical Ventilation in Emergency Medicine, https://doi.org/10.1007/978-3-319-98410-0}

The affiliation of Ani Aydin has been changed as following in the Front matter

Ani Aydin

Department of Emergency Medicine

Yale University School of Medicine

SkyHealth and Adult Ground Critical Care Transport

Yale-New Haven Health

New Haven, CT

USA 